\title{
Imprinting of ducklings to a second stimulus when a previously imprinted stimulus is occasionally presented*
}

\author{
LEONARD A. EISERER and HOWARD S. HOFFMAN \\ Bryn Mawr College, Bryn Mawr, Pennsylvania 19010
}

\begin{abstract}
Newly hatched ducklings were imprinted to one stimulus and then given prolonged visual exposure to a second discriminable stimulus. Filial behavior toward the second stimulus developed more slowly when the original imprinting stimulus was occasionally presented during exposure to the second stimulus than when the original stimulus was withheld during such exposure. Results were interpreted in terms of a possible motivational aftereffect following presentations of the original imprinting stimulus.
\end{abstract}

After imprinting to a moving stimulus, 5-day-old ducklings will gradually begin to emit filial behavior to a second stimulus if their exposure to it persists long enough (Hoffman, Ratner, \& Eiserer, 1973). However, in previous studies that have demonstrated the gradual emergence of filial behavior in older ducklings, exposure to the original stimulus was precluded during exposure to the second stimulus. The present research sought to determine the effect of intermittent exposure to the original imprinting stimulus during prolonged exposure to a second stimulus.

One possible finding is facilitation of the emergence of filial behavior toward the second stimulus via some positive transfer effect from the original stimulus to the second stimulus. It has been shown, for instance, that 5-day-old ducklings that have been previously imprinted to one stimulus show filial behavior toward a second unfamiliar stimulus more readily than do 5-day-old ducklings that have not been previously imprinted to any stimulus (Hoffman et al, 1973). This facilitation could be interpreted as suggesting that the imprinting attachment can somehow transfer from the first stimulus to the second, in which case the effect might be further enhanced if the two stimuli were experienced in temporal proximity, as in the design of the present experiment.

Alternatively, intermittent exposure to the original stimulus may, perhaps by maintaining specificity of the sort traditionally attributed to imprinting attachments (Lorenz, 1937; Thorpe, 1956), retard development of filial behavior toward the second stimulus. It is possible, of course, that under such conditions filial behavior toward the second stimulus would never develop.

The research reported here evaluated these possibilities by arranging to occasionally present the original imprinting stimulus to older, previously imprinted ducklings during a period when they were experiencing prolonged exposure to a second, visually distinctive imprinting-type stimulus. By assessing the

\footnotetext{
*This research was supported by NIMH Grant No. 19715. directed by the second author. Requests for reprints should be sent to Howard S. Hoffman, Department of Psychology, Bryn Mawr College, Bryn Mawr, Pennsylvania 19010.
}

development of filial behavior to the new stimulus and comparing it to the development of such behavior in a second group of previously imprinted ducklings treated similarly but denied the brief exposures to the original stimulus, it would be possible to determine the behavioral consequences of those exposures.

\section{METHOD}

\section{Subjects}

The Ss were 10 Khaki Campbell ducklings (Anas platyrhynchos domesticus), hatched in a forced-air incubator from eggs obtained from George F. Shaw, Inc., West Chester, Pennsylvania. When a given egg exhibited pip marks, it was transferred to a small plastic container $(15 \times 15 \times 15 \mathrm{~cm})$ with holes along the bottom and top for ventilation. These holes admitted light and sound and permitted visual inspection of the egg, but their arrangement prevented the duckling from viewing anything other than the interior of the container.

Ducklings were housed in individual 57-liter containers constructed of white translucent plastic and lined with a clear heavy-duty polyethylene bag. Absorbent material (Sanicel) was spread on the bottom of the bag. Food and water were continuously available from disposable plastic cups that were mounted on an aluminum support that hung inside the bag.

Except for the procedures of the experiment, the visual experience of the Ss was minimal. Having been exposed to only the interior of the hatching container and the translucent housing unit, the ducklings had no contact with moving stimuli.

\section{Apparatus}

The imprinting apparatus is illustrated in Fig. 1. It consisted of a plywood box $(122 \times 76 \times 76 \mathrm{~cm})$ divided by a fine mesh stainless steel screen into two compartments: a stimulus compartment $(122 \times 30 \times 76 \mathrm{~cm})$ and a $S$ compartment $(122 \times 46 \times 76 \mathrm{~cm})$.

The floor of the S compartment was covered with a thin layer of Sanicel. Lighting in this compartment was provided by two continuously illuminated $75-\mathrm{W}$ incandescent lamps mounted above the screen. These lamps were positioned so that, unless the stimulus compartment was also illuminated, light reflected from the stainless steel screen and prevented the $S$ from viewing into the stimulus compartment.

The stimulus compartment was divided into two equal sections by means of a vertical opaque barrier mounted perpendicular to the screen. The stimulus on one of these sections was a white rectangular foam-rubber object $(30 \times 10 \times 10 \mathrm{~cm})$ mounted over a model train engine. Presentations of this stimulus were produced by switching on an 


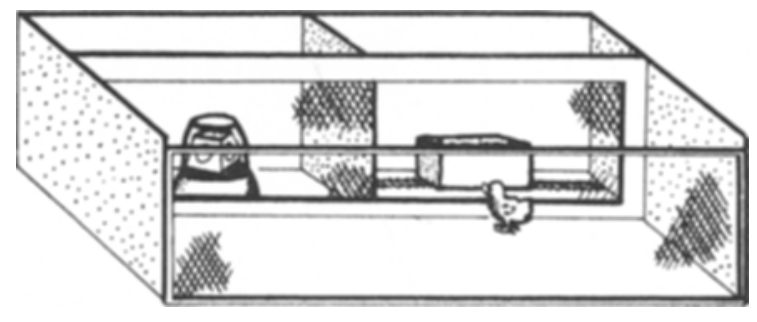

Fig. 1. Apparatus used for imprinting and testing. The stimulus on the left is the rotating lamp and the stimulus on the right is the moving object.

overhead $75-\mathrm{W}$ incandescent lamp in this section of the stimulus compartment and moving the stimulus back and forth along HO-gauge track at approximately $30 \mathrm{~cm} / \mathrm{sec}$. Stimulus withdrawal was accomplished by extinguishing the overhead lamp and stopping the movement of the stimulus.

The other section of the stimulus compartment contained a rotating amber-colored lamp of the sort used on the top of many police vehicles and ambulances. This lamp was dimmed by placing a translucent covering over the bulb and, by using the appropriate circuitry, it was arranged that its direction of rotation would reverse at approximately the same rate as that at which the moving foam-rubber stimulus changed direction (i.e., every $2 \mathrm{sec}$ ). Presentation of the rotating lamp was accomplished by illuminating its bulb and concurrently initiating rotation. With these stimuli and with the lighting arranged in this fashion, the $E$ could rapidly present or withdraw either or both of the stimuli as needed.

To permit the assessment of the ducklings' locomotor behavior during testing, a set of photocell units was used to divide the $S$ compartment into four quadrants (each measuring $61 \times 23 \mathrm{~cm}$ ). Two of the quadrants were directly in front of the screen that separated the $S$ from the stimuli. One of these corresponded in position to the portion of the stimulus compartment that contained the moving stimulus and the other corresponded to the portion that contained the rotating lamp. The other two quadrants also corresponded in position with the stimuli but were situated in the half of the $S$ compartment farthest from the screen. With this arrangement, it was possible to document the amount of time a given duckling spent in the immediate proximity of each stimulus during its presentation.

A bank of relays, stepping switches, and timers was employed to program stimulus presentation, and an operations recorder was used to document stimulus events as well as the position of the $\mathrm{S}$. In addition, the Ss were more or less continuously observed by means of a closed-circuit television system.

\section{Procedure}

After hatching, each duckling remained visually isolated in the incubator for 8-12 h. It was then transferred to the test apparatus, and its experimental sessions were begun. Each duckling was placed in its individual housing unit during the intervals between sessions.

The ducklings were divided into two groups of five birds each. Both groups received identical imprinting procedures over the first 3 days posthatch. These procedures consisted of six $20-\mathrm{min}$ sessions of continuous exposure to the foam-rubber moving stimulus. Two such sessions were run per day.

After the last imprinting session on Day 3 , each duckling remained in an individual housing unit until Day 5 , when the next phase of the experiment began. At the start of this phase, each duckling in both groups received an initial approach test with the novel rotating lamp. This test consisted of $2 \frac{1}{2} \mathrm{~min}$ of continuous exposure to the lamp, during which the position of the duckling was recorded. After this initial test, each duckling received six exposure sessions at the rate of two per day. During these sessions, the rotating lamp was presented for an equal amount of time to Ss in both groups. The two groups differed, however, with respect to whether the imprinted moving stimulus was occasionally present during exposure to the rotating lamp.

During the sessions for Group 1, the rotating lamp was initially presented continuously for $4 \mathrm{~min}$, followed immediately by a $30-\mathrm{sec}$ presentation of the imprinted moving stimulus. This sequence of stimulus presentation was repeated once and then, after a minute of complete stimulus withdrawal (i.e., neither stimulus was presented), a $2 \frac{1}{2}-$-min approach test was given with the rotating lamp. After this time, the session terminated and the duckling was returned to its housing unit. Sessions for Group 2 were identical to those for Group 1, except that during the two 30-sec periods of presentation of the original imprinted stimulus, ducklings in Group 2 received periods of complete stimulus withdrawal.

\section{RESULTS}

During all sessions, each duckling in Group 1 positioned itself in the quadrant nearest the original imprinting stimulus whenever that stimulus was presented. In contrast, all ducklings in both groups emitted fear-type responses (distress calling, flight to a far corner of the apparatus) when the novel rotating lamp was initially presented in Session 1. In later sessions, these responses diminished in frequency and magnitude, and the ducklings began to approach and stay near the rotating lamp when it was presented. Although by Session 6 both groups of ducklings were spending most of their time in the quadrant nearest the rotating lamp during its presentation, the two groups differed in the rate at which such filial behavior developed.

Figure 2 shows the mean percentage of time each group of ducklings spent in the quadrant nearest the rotating lamp after various intervals of exposure to that stimulus. Except for the initial position test, the data are plotted as averages of pairs of tests, so that the means represent the approach that occurred with each additional $16 \mathrm{~min}$ of exposure. Inspection of the figure reveals that, while $S s$ in both groups eventually came to station themselves near the lamp, the response developed more slowly in the group that had intermitteni presentations of the original imprinting stimulus (Group 1) than in the group that had no such presentations (Group 2).

The reliability of these effects was assessed through a repeated-measures analysis of variance (Winer, 1962, p. 302). This analysis yielded a significant sessions effect $(\mathrm{F}=24.4, \mathrm{df}=3 / 24, \mathrm{p}<.05)$, a significant interaction of Groups by Sessions $(F=3.14, d f=3 / 24, p<.05)$, and an insignificant overall difference between groups $(F=3.68, d f=1 / 8, p>.05)$. Since this analysis yielded a significant interaction term, tests on simple main effects (Winer, 1962, p. 303) were performed to assess the observed differences between the two groups after the various amounts of exposure to the second stimulus. These tests indicated that the large observed differences at 16 and $32 \mathrm{~min}$ were statistically significant $(F \geqslant 6.97$, $\mathrm{df}=1 / 8, \mathrm{p}<.05$ in each case) but that the smaller 
differences at 0 and $48 \mathrm{~min}$ were not. Taken together, this configuration of statistical results is exactly what would be expected if, as implied by the averages depicted in Fig. 2, intermittent exposure to a previously imprinted stimulus during exposure to a second novel stimulus retarded but did not preclude the development of filial behavior toward the second stimulus.

\section{DISCUSSION}

The present study has demonstrated that intermittent visual contact with the original imprinting stimulus can retard the development of filial behavior toward a second stimulus. These results are consistent with the finding of Guiton (1959) and of Sluckin and Salzen (1961) that previously socialized (i.e., group-reared and mutually imprinted) chicks that are isolated before exposure to an unfamiliar moving object show stronger filial behavior toward that object than socialized chicks that are not subsequently isolated. However, in those studies, the socialized-then-isolated Ss received considerably less social rearing (and hence less exposure to the original imprinting stimuli) than the socialized-only Ss. Consequently, the latter group may have shown less filial behavior toward the second stimulus either because of more recent exposure to the original stimulus or because of greater amount of imprinting experience with the original stimulus.

The present experiment, in which ducklings in both groups were virtually equivalent in amount of exposure to the original stimulus ( 120 vs $126 \mathrm{~min}$ ), suggests that recency of contact with the original stimulus relative to exposure to the second stimulus is a critical factor in determining the rate at which filial behavior toward the second stimulus develops. Of course, amount of initial exposure to the first stimulus may also affect this rate.

While the present experiment does not permit specification of the exact process underlying this recency effect, one reasonable possibility stems from the strong behavioral aftereffects that have been found to follow brief presentation of an imprinting stimulus (Eiserer \& Hoffman, 1973). These aftereffects-which include both an enhancement of distress vocalization and an enhancement of an operant response previously reinforced by presentation of the imprinting stimulus-have been interpreted to represent an increase in the motivation of the ducklings for additional stimulation by the imprinting object. In the present study, it is possible that for ducklings in Group 1 each of the occasional presentations of the original imprinting stimulus was followed by a motivating aftereffect that was specifically directed toward the nonwithdrawn original imprinting stimulus. These aftereffects, absent during the sessions of Group 2, may have interfered with the development of filial behavior toward the second stimulus.

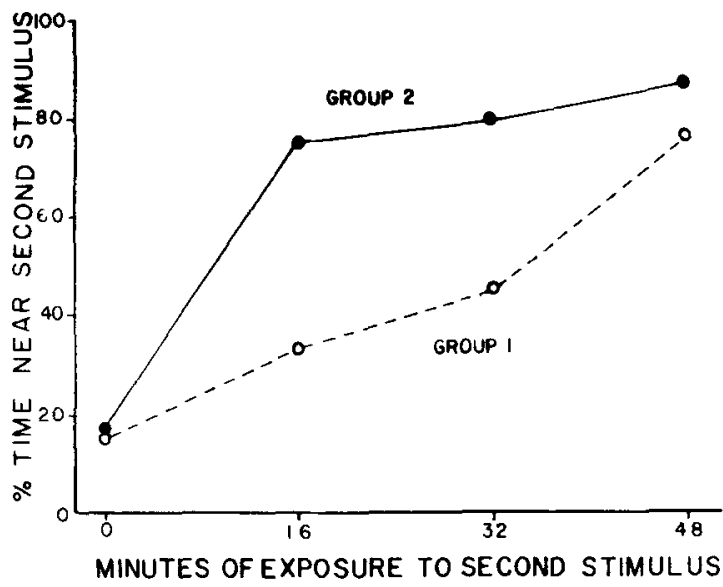

Fig. 2. Percent of time spent in the quadrant nearest the second stimulus as a function of minutes of exposure to that stimulus.

In any event, the present results, together with those of Hoffman et al (1972), suggest a complex effect of prior imprinting on the formation of new social bonds. As shown by Hoffman et al, a history of prior imprinting to one stimulus facilitates subsequent formation of social attachment to a second and distinct stimulus, relative to a history of no previous imprinting. However, as found in the present experiment, this facilitation can be reduced if intermittent exposure to the previously imprinted stimulus occurs during the period of exposure to the second stimulus.

One other point is worth making concerning the results of the present study. As already noted, ducklings in Group 1 continued to approach and station themselves near the original imprinting stimulus whenever that stimulus was presented, even during the last few sessions when approach to the second stimulus was strongly developed. In other words, these ducklings showed no weakening of their attachment to the original imprinting stimulus as they developed an attachment to the second stimulus.

\section{REFERENCES}

Eiserer, L. A., \& Hoffman, H. S. Priming of ducklings' responses by presenting an imprinted stimulus. Journal of Comparative \& Physiological Psychology, 197 3, 82, 345-359.

Guiton, $P$. Socialization and imprinting in Brown Leghorn chicks. A nimal Behaviour, 1959, 7, 26-34.

Hoffman, H. S., Ratner, A. M. \& Eiserer, L. A. Role of visual imprinting in the emergence of specific filial attachments in ducklings. Journal of Comparative \& Physiological Psychology, 1972, 81, 399-409.

Lorenz, $K$. The companion in the bird's world. Auk, 1937, 54, 245-273.

Sluckin, W., \& Salzen, E. A. Imprinting and perceptual learning. Quarterly Journal of Experimental Psychology, 1961, 13, 65-77.

Thorpe, W. H. Learning and instinct in animals. London: Methuen, 1956. 2nd ed, 1963.

Winer, B. J. Statistical principles in experimental design. New York: McGraw-Hill, 1962.

(Received for publication November 16, 1973; revision received January 22,1974 .) 